A NEW TEST OF COMPOUND SYMMETRY

\title{
by
}

\author{
S. N. ROY \\ INSTITUTE OF STATISTICS, UNIVERSITY OF NORTH CAROLINA
}

Institute of Statistics

Mimeograph Series No. 97

March 11, 1954

1 Work sponsored by the Office of Naval Research under Contract No. N7-onr-28402. 


\section{A NEW TEST OF COMPOUND SMMETRY}

by

\section{S. N. ROY}

Institute of Statist1cs, University of North Carolina

\section{Summary. If $x_{1}$ and $x_{2}$ have a bivariate normal distribution with}

a correlation coefficient $\rho$ and the same standard deviations $\sigma$ for both, then it is well known and easy to check that $x_{1}+x_{2}$ and $x_{1}-x_{2}$ are uncorrelated, which forms the basis of Pitman's well-known test of $\mathrm{H}_{0}: \sigma_{1}=\sigma_{2}$, for a bivariate normal population, in terms of the correlation coefficient $r$ between $x_{1}+x_{2}$ and $x_{1}-x_{2}$ in a random sample of size, say, $n$, from this population. Starting from this test which has a number of reasonably good properties, and then using the union-intersection principle $[1,2]$, a test is obtained for compound symmetry, $1 . e .$, for $\mathrm{H}_{0}: \sigma_{11}=\sigma_{22}=\ldots=\sigma_{p p}$ and all $\sigma_{1 j}$ 's are equal ( $\left.1 \neq j=1,2, \ldots, p\right)$, where $\sigma_{1 j}$ is any element of the covariance matrix $\Sigma$ of a p-variate normal population. 2. Test Construction. Let $\rho\left(x_{1}, x_{2}\right)$ denote the population correlation coefficient between $x_{1}$ and $x_{2}$ and $r\left(x_{1}, x_{2}\right)$ the same in a random sample of size, say $n$, from that population. Then, for a set of stochastic variables $\underline{x}^{\prime}(1 \times p)\left(=x_{1}, \ldots, x_{p}\right)$ having a p-variate normal distribution, notice that for any arbitrary non-null vector $\underline{a}^{\prime}(1 \times p), \underline{a}^{\prime}(1 \times p) \underline{x}(\mathrm{p} \times l)$ and $\sum_{i=1}^{p} x_{i}$ have a bivariate normal distribution and, now letting $\sum_{i=1}^{p} a_{i}=0$, 
consider the hypothesis: $\rho\left(\sum_{i=1}^{p} x_{1}, \underline{a}^{\prime} \underline{x}\right)=0=\mathrm{H}_{\mathrm{Oa}_{\mathrm{a}}}$ (say). Next notice that

(2.1) $H_{0}$ : all $\sigma_{i j}$ 's are equal and all $\sigma_{i j}$ 's are equal $(i \neq j=1, \ldots, p)$

$=\bigcap_{\underline{a}{ }^{H} \underline{a}}=\bigcap_{\underline{a}}\left[\rho\left(\sum_{i=1}^{p} x_{1}, \underline{a}^{\prime} \underline{x}\right)=0\right]$,

where $\underline{a}^{\prime}(1 \times \mathrm{l} \quad \mathrm{p})$ is any arbitrary row vector subject to $\sum_{i=1}^{p} a_{1}=0$.

Now going back to $\mathrm{H}_{\mathrm{Oa}}$, we have for this hypothesis the Pitman critical region, say $W_{\underline{a}}(\alpha)$, of size $\alpha$, given by

$$
W_{\underline{a}}(c): \quad r^{2}\left(\sum_{i=1}^{p} x_{i}, \underline{a}^{\prime} \underline{x}\right) \geq r_{c}^{2}(n-2),
$$

where $r_{c}(n-2)$ is the upper $\alpha / 2$-point of the central $r$-distribution in random samples of size $n$.

Hence, by the union-intersection heuristic principle $[1,2]$ we have, for $\mathrm{H}_{\mathrm{O}}\left(=\bigcap_{\underline{\mathrm{a}}} \mathrm{H}_{\mathrm{Oa}}\right)$, the critical region $\mathrm{W}(\beta)$ of size $\beta$ given by

$$
\begin{aligned}
& w(\beta): \bigcap_{a}\left[r^{2}\left(\sum_{i=1}^{p} x_{1}, \underline{a}^{\prime} \underline{x}\right) \geq r_{a}^{2}(n-2)\right], \\
& \text { i.e., } \sup _{\underline{a}} r^{2}\left(\sum_{i=1}^{p} x_{1}, \underline{a}^{\prime} \underline{x}\right) \geq r_{a}^{2}(n-2) \\
& \text { 1.e., } \sup _{\underline{a}} r^{2}\left(\sum_{i=1}^{p} x_{i}, \underset{i=1}{p-1} a_{i}\left(x_{i}-x_{p}\right)\right) \geq r_{c}^{2}(n-2),
\end{aligned}
$$

remembering that $\sum_{1=1}^{p} a_{i}=0$, i.e., $a_{p}=-\sum_{i=1}^{p-1} a_{i}$. 


\section{$-3-$}

It is easy to check that

$$
\sup _{\underline{a}} r^{2}\left(\sum_{i=1}^{p} x_{1}, \sum_{i=1}^{p-1} a_{1}\left(x_{i}-x_{p}\right)\right)
$$

= square of the sample multiple correlation between $\sum_{i=1}^{p} x_{1}$ and the $(p-1)$ set of variables $\left(x_{1}-x_{p}\right),\left(x_{2}-x_{p}\right), \ldots,\left(x_{p-1}-x_{p}\right)=$ $R^{2}\left[\sum_{i=1}^{p} x_{i}\right.$ and $\left.\left(x_{1}-x_{p}\right),\left(x_{2}-x_{p}\right), \ldots,\left(x_{p-1}-x_{p}\right)\right]=R^{2}$ (say). Notice that, since the new p-set also has the p-variate normal distribution, this $R$ has the well-known multiple correlation distribution with degrees of freedom $p-I$ and $n-p$ and a non-centrality parameter which is the population multiple correlation between $\sum_{1=1}^{p} x_{1}$ and the $(p-1)$-set above and which let us call $\rho^{2}$. It is easy to check that $\rho=0,1 . e$. , that $R$ has the central multiple correlation distribution, if and only if $p\left(\sum_{i=1}^{p} x_{i}, x_{1}-x_{p}\right)=0(i=1,2, \ldots, p-1)$, i.e., if and only if $\rho\left(\sum_{i=1}^{p} x_{i}, \underline{a}^{\prime} \underline{x}\right)=0$ (for all non-null a subject to $\sum_{i=1}^{p} a_{1}=0$ ). We have thus, for testing compound symmetry, the critical region $W(\beta)$ of size $\beta$ given by (2.5) $R\left[\sum_{i=1}^{p} x_{i}\right.$ and $\left.\left(x_{1}-x_{p}\right), \ldots,\left(x_{p-1}-x_{p}\right)\right] \geq R_{B}(p-1, n-p)$, when $R_{\beta}$ is the upper $\beta$-point of the well-known central multiple correlation distribution. 


\section{$-4-$}

It can be checked after some little algebra that in terms, respectively, of the elements of the sample and population covariance matrices $S$ and $\Sigma$, $R$ and $\rho$ will be given by

$$
\begin{aligned}
& R^{2}=1-p \sum_{i=1}^{p} z_{i} z^{1} /\left(\sum_{i=1}^{p} z_{i}\right)\left(\sum_{i=1}^{p} z^{1}\right), \\
& p^{2}=1-p \sum_{i=1}^{p} \zeta_{i} \zeta^{i} /\left(\sum_{i=1}^{p} \zeta_{i}\right)\left(\sum_{i=1}^{p} \zeta^{i}\right),
\end{aligned}
$$

when

$$
\begin{aligned}
& z_{1}=\sum_{j=1}^{p} s_{i j}, \quad z^{i}=\sum_{j=1}^{p} s^{i j}, \\
& \zeta_{1}=\sum_{j=1}^{p} \sigma_{i j} \text { and } !^{i}=\sum_{j=1}^{p} \sigma^{i j} .
\end{aligned}
$$

Note that $s_{i j}=s_{j 1}, s^{1 j}=s^{j i}, \sigma_{i j}=\sigma_{j 1}$ and $\sigma^{i j}=\sigma^{j 1}$.

The power properties of this test will be discussed in a later note.

\section{$\underline{\text { References }}$}

1. S. N. Roy, "On a heuristic method of test construction and its use in multivarlate analysis," annals of Mathematical Statistics, Vol. 24 (1953), pp. 220-38.

2. S. N. Roy and R. C. Bose, "Simultaneous confidence interval estimation," Annals of Mathematical Statistics, Vol. 24 (1953), pp. 513-36. 